\title{
Influence of Different Organoclays on the Curing, Morphology, and Dynamic Mechanical Properties of an Epoxy Adhesive
}

\author{
M. A. GARCÍA DEL CID ${ }^{1}$, M. G. PROLONGO ${ }^{1}$, C. SALOM ${ }^{1}$, \\ M. SÁNCHEZ-CABEZUDO ${ }^{2}$, and R. M. MASEGOSA ${ }^{2}$ \\ ${ }^{1}$ Departanento de Materiales y' Producción Aercespacial, Unitersidad Politécnica \\ de Madrid, Madrid, Spain \\ ${ }^{2}$ Depantamento de Fisica y Quinica Aplicadas a la Técnica Aeronántica, Unitersidad \\ Politénica de Madrid, Madrid, Spain
}

The thermal, mechanical, and adbesive properties of nanoclaymodified adbesives were investigated. Tuo organically modified montmorillonites: Cloisite $93 \mathrm{~A}$ (C93A) and Nanomer I.3OE (I.3OE) were used as reinforcement of an epoxy adbesive. C93A and I.3OE are modified with tertiary and primary alkyl ammoninm cations, respectively. The aim was to study the influence of the organoclays on the curing, and on the mechanical and adbesive properties of the nanocomposites. A specific goal uas to compare their bebavior with that of Cloisite30B/epoxy and Cloisite15A/ epoxy nanocomposites that we have previously studied. Both $C 3 O B$ and $C 15 A$ are modified with quaternary alkyl ammonium cations. Differential scanning calorimetry results shoued that the clays accelerate the curing reaction, an effect that is related to the chemical structure of the ammonium cations. The three Cloisite/nanocomposites shoued intercalated clay structures, the interlayer distance was independent of the clay content. The I.3OE/epoxy nanocomposites presented exfoliated structure due to the catalytic effect of the organic modifier. Clay-epoxy nanocomposites shoued louer glass transition temperature $\left(T_{g}\right)$ and bigher values of storage modulus than neat epaxy thermoset, with no 
significant differences between exfoliated or intercalated nanocomposites. The shear strength of aluminum joints using clay/epoxy adbesives was lower than with the neat epoxy adbesive. The water aging was less damaging for joints with I.3OE/epoxy adbesive.

\section{KEYWORDS Adbesives with nanoparticles; Aging; Dynamic mechanical analysis; Epoxy/epoxides; Lap-shear; Thermal analysis}

\section{INTRODUCTION}

Epoxy resins after the crosslinking (curing) give rise to thermosetting materials. These polymers are widely used as coatings, composite matrices, and adhesives. The reason lies in their excellent properties, combining creep resistance and rigidity, mechanical and thermal resistance, environmental stability, good chemical resistance, electrical insulating performance, and high adhesive strength. Other attractive features are the absence of volatile and low shrinkage on curing. Epoxy resins are versatile and can be crosslinked in a broad range of temperatures and with different degrees of crosslinking. The curing agents used at room temperature are usually aliphatic amines, however curing with aromatic amine needs high temperatures. In general, epoxy cured at high temperature exhibits improved high temperature properties such as increased glass transition temperature $\left(T_{g}\right)$, higher strength, and stiffness as compared to those cured at room temperature [1]. Epoxies are used as structural adhesives, matrices for composite materials, encapsulating agents, etc.

Epoxy/clay nanocomposites are materials obtained by dispersing clay platelets in epoxy thermosets. Montmorillonite is the most commonly used layered silicate in polymer matrix nanocomposites. In order to obtain a good dispersion of the clay layers in the polymer, the clays are modified by replacing the inorganic cations (located between the silicate layers) by alkyl ammonium cations [2-5]. Clay/epoxy nanocomposites have been investigated in recent years [6-22], because it is expected that the homogeneous dispersion of clay layers in the polymer leads to improved mechanical and thermal barrier properties compared to the pure polymer and to conventional composites with similar reinforcement content.

In the nanocomposite the epoxy monomers intercalate between the clay layers, leading to an increase of the clay spacing and, in the best of cases to the exfoliation of the silicate layers that become dispersed in the polymer matrix. The epoxy curing reaction occurs inside and outside the clay galleries (space between silicate layers). It has been proposed that the exfoliation depends on the ratio between the rates of both reactions [7]. Exfoliated clay/epoxy nanocomposites are obtained using modified clays with primary and secondary alkyl ammonium cations $[6,8,12]$, whereas when modified clays with quaternary alkyl ammonium are used most research indicate the formation intercalated nanocomposites $[16,18,21]$. 
The correlation of the improvement in mechanical and thermal properties with morphology (intercalated or exfoliated) of clay/epoxy nanocomposites is not completely understood yet. In low $T_{g}$ epoxy nanocomposites, specifically those cured with aliphatic diamines, improvements in modulus have been found for both exfoliated intercalated morphologies [6,8,10,16-19]. On the other hand, the effect of dispersion of clay in rigid epoxy thermosets which have a high $T_{g}$ (cured with aromatic diamines) is not clear, both increases and decreases in $T_{g}$ have been reported $[11-13,16,18,19]$.

Epoxy thermosets have excellent adhesive performance provided the service temperature is lower than their $T_{g}$. This is because the mechanical properties of the adhesive, such as the elastic modulus, decrease drastically in the vicinity of $T_{g}$. At that temperature the adhesive starts to lose the ability to transfer stresses. Few studies of resistance of adhesive bonds using nanoclay reinforced epoxy adhesives (nanocomposites) have been reported. Recently Al-Safy and collaborators [23] have studied the properties of low $T_{g}$ epoxy adhesive modified with an organoclay and used for concretecomposite joints.

In the present work the influence of two organically modified montmorillonite (Closite 93A and Nanomer I.30E) on the curing and thermal and dynamic mechanical properties of a high $T_{g}$ epoxy adhesive is studied. The objective is to compare the properties of I.30E/epoxy and C93A/epoxy nanocomposites with the properties of $\mathrm{C} 15 \mathrm{~A} /$ epoxy and $\mathrm{C} 30 \mathrm{~B} / \mathrm{epoxy}$ nanocomposites previously studied [18], to correlate their behavior with the chemical structure of the alkyl ammonium cations and with the intercalation of the epoxy adhesive in the clays. Further, the shear strength of adhesive joints using these clay/epoxy adhesives was studied, for freshly prepared and water aged joints.

\section{EXPERIMENTAL}

\subsection{Materials}

The epoxy monomer used was diglycidyl ether of bisphenol A (DGEBA) with commercial name of Araldit F (Huntsman, Basel, Switzerland). The number average relative molecular mass, $\bar{M}_{n}=380 \mathrm{~g} \cdot \mathrm{mol}^{-1}$, was obtained by chemical titration of the end groups. The curing agent was $4,4^{\prime}$ diaminodiphenylmethane (DDM) $(97 \mathrm{wt} \%)$ supplied by Sigma-Aldrich Chemical Co. (Buchs, Switzerland). The organoclays used were: Cloisite 93A (C93A) (Southem Clay Products, Inc., Gonzales, TX, USA) and Nanomer I.30E (Nanocor Inc., Chicago, IL, USA). The characteristics of C93 and of I.30E are given in Table 1, that is, alkyl ammonium cation (surfactant), cation exchange capacity (CEC), and basal spacing $\left(d_{001}\right)$. Table 1 also collects the characteristics of Cloisite $30 \mathrm{~B}$ and Cloisite $15 \mathrm{~A}$. The interlayer spacing $\left(d_{001}\right)$ listed in Table 1 was obtained from our experiments of wide-angle X-ray diffraction (WAXD) as it is described in Sec. 2.5. The Cloisites have a moisture 
TABLE 1 Characteristics of the Organically Modified clays

\begin{tabular}{|c|c|c|c|}
\hline Clay & Alkyl ammonium cations & $\begin{array}{c}\text { CEC } \\
\text { (meq/100g) }\end{array}$ & $\begin{array}{l}d_{001} \\
\text { (nim) }\end{array}$ \\
\hline $\mathrm{C} 93 \mathrm{~A}$ & Methyl, di(hydrogeneted talow*) onium & 90 & 2.56 \\
\hline C30B & Methyl, tallow ${ }^{*}$ di-2-hydroxyethyl quaternary ammonium & 90 & 1.82 \\
\hline C15A & Dimethyl, dihydrogenated tallow*, quaternary ammonium & 125 & 3.15 \\
\hline $\mathrm{I} .30 \mathrm{E}$ & Octadecyl ammonium & 95 & 2.19 \\
\hline
\end{tabular}

content less than $2 \mathrm{wt} \%$. Loctite $44 \mathrm{NC}$ Frekote (Henkel, Düsseldorf, Germany) was employed as mold release product.

\subsection{Preparation of Clay Dispersions and Nanocomposites}

Dispersions of C93A and of I.30E in DGEBA were prepared, by adding the clay powders to the epoxy monomer, stirring at $300 \mathrm{rpm}$ at $120^{\circ} \mathrm{C}$ for 2 hours. After that, the dispersions were degassed for about 15 minutes under vacuum at $90^{\circ} \mathrm{C}$. Then the curing agent (DDM) was added in amino-hydrogen/epoxy stoichiometric ratio and mixed at $80^{\circ} \mathrm{C}$ with stirring for 5 minutes. Finally the dispersion was sonicated for 2 minutes. The prepared dispersions had different clay content: $0,2,4,6,10$, and $12 \mathrm{wt} \%$. Small amounts (10-20 $\mathrm{mg}$ ) of these dispersions were poured into aluminum pans and scanned in the differential scanning calorimeter (DSC). The dispersions were poured into aluminum molds, whose walls were previously treated with mold release product, for curing in an oven and the nanocomposites were obtained. The curing protocol used was: $120^{\circ} \mathrm{C}$ for 1 hour, then the temperature was raised to $180^{\circ} \mathrm{C}$ and kept at $180^{\circ} \mathrm{C}$ for 2 hours. The curing was carried out under atmospheric pressure. Specimens having dimensions $35 \times 10 \times 2 \mathrm{~mm}^{3}$ were cut for dynamic mechanical thermal analysis (DMTA).

\subsection{Differential Scanning Calorimetry}

A DSC (Mettler Toledo mod.822e, Greifensee, Switzerland) was used to measure the glass transition temperature $\left(T_{g}\right)$ and heat of reaction $(\Delta H)$. The instrument was calibrated with indium and zinc and the measurements were registered at a heating rate of $10^{\circ} \mathrm{C} / \mathrm{min}$ under nitrogen atmosphere. A M4 Sartorius microbalance (Sartorius, Göttingen, Germany) was used to weigh the samples of about $10-20 \mathrm{mg}( \pm 0.01 \mathrm{mg})$. The $T_{g}$ was taken at the midpoint of the heat capacity change.

\subsection{Dynamic Mechanical Thermal Analysis}

DMTA of cured samples was performed in dual cantilever bending mode using a DMTA V instrument (Rheometric Scientific, Piscataway, NJ, USA). Measurements were done at $1 \mathrm{~Hz}$, with temperature increasing from $30^{\circ} \mathrm{C}$ 
to $220^{\circ} \mathrm{C}$ at a heating rate of $2^{\circ} \mathrm{C} \mathrm{min}{ }^{-1}$. The elastic or storage modulus $\left(E^{\prime}\right)$, the loss modulus $\left(E^{\prime \prime}\right)$, and $\tan \delta$ isochrones were recorded as a function of temperature. The maxima in $\tan \delta$-temperature plots were determined to identify the $\alpha$-relaxations associated to the glass transitions.

\subsection{WAXD and Transmission Electron Microscopy}

The intercalation of the epoxy in the clays galleries was studied by WAXD and Transmission Electron Microscopy (TEM). WAXD patterns were obtained at room temperature in reflection mode using a Panalytical X'Pert PRO Alpha1 diffractometer (PANalytical BV, Eindhoven, The Netherlands) equipped with a curved Ge111 primary beam monochromator and a fast detector X'Celerator (Cu K $\alpha 1$ radiation, $\lambda=0.15406 \mathrm{~nm}, 45 \mathrm{kV}, 40 \mathrm{~mA}$ ). The measurement range of $2 \Theta$ was from $1.5^{\circ}$ to $40^{\circ}$, and step size was $0.0167^{\circ}$.

Cryomicrotomy cutting was used to prepare samples for TEM. The films were coated with a thin layer of Au (Pd). The TEM equipment was a Philips Tecnai $20 \mathrm{~T}(200 \mathrm{kV})$ (Philips, Eindhoven, The Netherlands) with SiS CCD camera model Keenview.

\subsection{Preparation of Adhesive Joints and Lap Shear Test}

The single lap adhesive joints are generally used for comparative studies of adhesives. To study the adhesive behavior of the organoclay/epoxy nanocomposites the strength of adhesive joints in single lap was determined following the UNE-EN-ISO 1465 standard. The aluminum substrates (alloy EN AW 5052) had dimensions: $100 \times 25 \times 2 \mathrm{~mm}^{3}$, their surfaces were treated following the sequence: Scotch-Brite ${ }^{\mathrm{B}}$ abrasion, rinsed with distilled water, degreasing with methyl ethyl ketone (MEK) at room temperature, rinsed with distilled water, alkaline dip $\left(100 \mathrm{gL}^{-1} \mathrm{NaOH}\right)$ at $60^{\circ} \mathrm{C}$ for 1 minute, rinsed with distilled water, acid-aging treatment immersion in an aqueous solution of $146 \mathrm{gL}^{-1}$ of $\mathrm{Fe}_{2}\left(\mathrm{SO}_{4}\right)_{3} \cdot 9 \mathrm{H}_{2} \mathrm{O}$, and $185 \mathrm{~mL} \cdot \mathrm{L}^{-1}$ of $\mathrm{H}_{2} \mathrm{SO}_{4}$. Finally, all samples were rinsed in distilled water and dried with hot air. This sulfuric acid-ferric sulfate based treatment is known as P2 [24,25]. After that the substrates were joined in single lap using a steel mold with a step that allows to obtain probes with controlled adhesive thickness. After curing the thickness of the lap shear probes was measured finding that the adhesive thickness was $0.30 \pm 0.10 \mathrm{~mm}$ and the dimensions of the lap joints were $25 \times 12.5 \mathrm{~mm}^{2}$. At least seven replicates for each adhesive composition were tested.

\section{RESULTS AND DISCUSSION}

\subsection{Dynamic Curing of Organoclay/DGEBA Dispersions by DSC}

The dispersions of the organoclays $(6 \mathrm{wt} \%)$ in DGEBA without adding the curing agent were scanned in the DSC to check the reactivity. 
Figure 1 shows the DSC thermograms for the dispersions and for DGEBA. The data of $\mathrm{C} 30 \mathrm{~B}$ and $\mathrm{C} 15 \mathrm{~A}$ are taken from our previous study [21].

In all cases a glass transition was observed, $T_{g}^{0} \sim-16^{\circ} \mathrm{C}$ (not shown in the figure), which corresponds to the unreacted DGEBA, it is followed by a single exothermic peak corresponding to the DGEBA homopolymerization [21]. This exothermic peak appears at lower temperatures in the dispersions showing the catalytic effect of the clays. It can be seen that I.30E favors homopolymerization more than $\mathrm{C} 30 \mathrm{~B}, \mathrm{C} 93 \mathrm{~A}$, and $\mathrm{C} 15 \mathrm{~A}$. This behavior indicates that the primary alkyl ammonium ions of $\mathrm{I} .30 \mathrm{E}$ are more effective in activating homopolymerization than the hydroxyl groups of the alkyl ammonium cations of the $\mathrm{C} 30 \mathrm{~B}$ or the non-polar groups of the alkyl ammonium cations of C15A. After the first scan, a second scan was performed from $0-300^{\circ} \mathrm{C}$, which showed only a glass transition around $88^{\circ} \mathrm{C}$ associated with the epoxy homopolymer (polyether) formed.

\subsection{Dynamic Curing of Organoclay/DGEBA-DDM Dispersions by DSC}

Figure 2 shows the thermograms for dispersions of C93A in DGEBA-DDM. A glass transition temperature $\left(T_{g}{ }^{0}\right)$ is observed corresponding to the unreacted mixture, it is followed by the exothermic peak corresponding to the DGEBA-DDM curing reaction. The addition of the clay shifts the exothermal peak to lower temperatures, therefore the curing reaction is accelerated in the presence of C93A. It has been reported that I.30E accelerates the curing reaction of the epoxy resin [15], to compare its effect

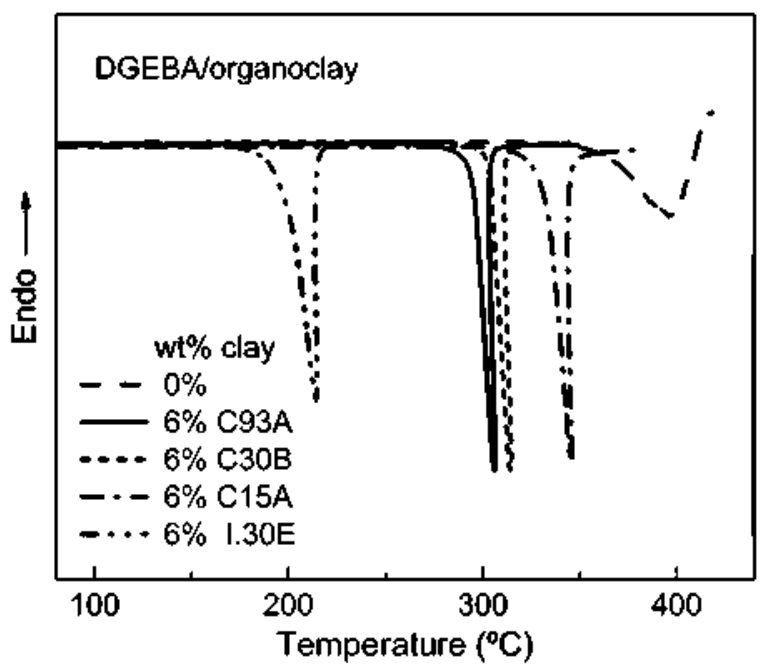

FIGURE 1 DSC scans of organoclay/DGEBA dispersions. The data of C30B and C15A are taken from our previous study [21]. 


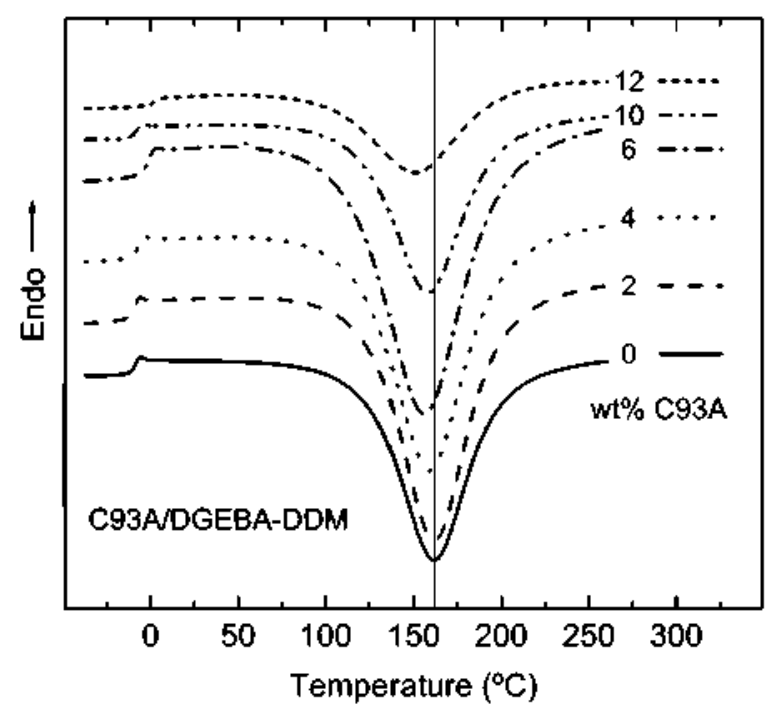

FIGURE 2 DSC scans of C93A/DGEBA-DDM dispersions.

with those of Cloisites under the same conditions the I.30E/epoxy dispersions have been studied by DSC.

The $T_{g}^{0}$ values, the exothermic peak temperature $\left(T_{\text {peak }}\right)$ and the heat released in the reaction $(-\Delta H)$ calculated from the peak area are given in Table 2. From the $T_{\text {peak }}$ values it is deduced that the curing reaction is accelerated more significantly by I.30E. The acidic alkyl ammonium cations catalyze the homopolymerization of both the epoxy and the epoxy-amine reaction [7-10]. The behavior observed for C93A/epoxy dispersions is similar to the observed in $\mathrm{C} 30 \mathrm{~B} / \mathrm{epoxy}$ dispersions [18]. The organic cation of $\mathrm{C} 93 \mathrm{~A}$

TABLE $2 T_{\mathrm{g}}^{\hat{y}}, T_{\text {peak }},-\Delta H$ (Referred to Epoxy Equivalent), and $T_{g}$ Obtained from the Dynamic Curing in the DSC at $10^{\circ} \mathrm{C} / \mathrm{min}$ for C93A/DGEBA-DDM and I.30E/DGEBA-DDM Dispersions

\begin{tabular}{|c|c|c|c|c|c|}
\hline \multirow[b]{2}{*}{$w t \%$} & \multirow[b]{2}{*}{$T_{g}^{n}\left({ }^{\circ} \mathrm{C}\right)$} & \multirow[b]{2}{*}{$T_{\text {peak }}\left({ }^{\circ} \mathrm{C}\right)$} & \multirow[b]{2}{*}{$-\Delta H\left(\mathrm{~J} / e e^{*}\right)$} & \multicolumn{2}{|c|}{$T_{g}(\mathrm{C})$} \\
\hline & & & & 2nd scan & 3rd scan \\
\hline \multicolumn{6}{|c|}{ C93A } \\
\hline 0 & -10 & 162 & 99 & 160 & 160 \\
\hline 2 & -10 & 162 & 95 & 156 & 156 \\
\hline 4 & -8 & 159 & 96 & 156 & 153 \\
\hline 6 & -1 & 156 & 96 & 151 & 149 \\
\hline 10 & -8 & 158 & 94 & 147 & 147 \\
\hline 12 & 2 & 151 & 84 & 142 & 144 \\
\hline \multicolumn{6}{|c|}{ I.30E } \\
\hline 4 & -1 & 149 & 82 & 144 & 145 \\
\hline 6 & 6 & 146 & 75 & 141 & 142 \\
\hline
\end{tabular}

•Eprosy equivalent weight. 
has one hydrogen and the organic cation of C30B has two hydroxyl groups which catalyze the epoxy-amine reaction and even the hydroxyls can react with epoxy groups. However this catalytic effect was not observed in C15A/epoxy dispersions [18], because in this clay the alkyl ammonium cations have no hydroxyl groups neither are acids (see Table 1).

The values of $-\Delta H$ (see Table 2 ) depend on the clay content, indeed for high clay content $(>10 \mathrm{wt} \%)$ the reaction becomes much less exothermic. Previous results for C30B/DGEBA-DDM system showed a similar trend, however the effect for C15A/DGEBA-DDM was less noticeable [18]. The variation of $-\Delta H$ with the clay content may be attributed to two factors: the nature of the alkyl ammonium cations that determines their participation in the curing reaction, and second to the different degree of intercalation of DDM and DGEBA molecules into the clay galleries which disturb the stoichiometry resulting smaller values of $-\Delta H$ [21]. The influence of this last factor is supported by the decrease of the $T_{g}$ of the epoxy thermoset in the presence of clay, as it will be discussed below. It is worth noting that curing with DDM the homopolymerization of DGEBA is unlikely since it occurs at temperatures $>300^{\circ} \mathrm{C}$ (see Fig. 1) when all epoxy groups have reacted with the amino groups [21]. However curing with DDM in presence of $\mathrm{I} .30 \mathrm{E}$ both reactions (amine-epoxy and the epoxy homopolymerization) can compete, this is another factor that could justify the different values $-\Delta H$ in I.30E/epoxy dispersions.

The glass transitions of the unreacted C93A/epoxy dispersions $\left(T_{g}^{0}\right)$ are higher than those of DGEBA-DDM, suggesting good interaction between C93A and the uncured resin. A similar behavior was found for the dispersions with C30B and C15A [18]. However for I.30E/DGEBA-DDM dispersion the $T_{g}^{0}$ increase is significantly higher, which reflects good interactions between the epoxy resin and I.30E.

Table 2 also shows the values of $T_{g}$ of the epoxy thermoset that was cured during the first DSC scan, these $T_{g}$ values were obtained from the second and third DSC scans. It can be seen that the $T_{g}$ of epoxy thermoset decreases with increasing the clay content. The effect is more pronounced for I.30E indicating that the resin homopolymerization, promoted by acid alkyl ammonium ions, leads to stoichiometric imbalances that result in a less crosslinked epoxy thermoset.

\subsection{Glass Transitions of Organoclay/Epoxy Nanocomposites by DSC}

First of all, the influence of the curing protocol on the C93A-DGEBA dispersions without curing agent was studied. Dispersions of C93A in DGEBA were prepared and heated in an oven following the curing protocol: 1 hour at $120^{\circ} \mathrm{C}$ followed by 2 hours at $180^{\circ} \mathrm{C}$, afterwards, they were DSC scanned. They did not show any change in $T_{g}^{0}\left(-16^{\circ} \mathrm{C}\right)$. Hence, under the curing conditions 
no reaction takes place between DGEBA and C93A. In the DSC scan an exothermic peak appears at high temperature $307^{\circ} \mathrm{C}$ that corresponds to the DGEBA homopolymerization. Similar behavior was found for $\mathrm{C} 30 \mathrm{~B}$, and C15A epoxy dispersions [18]. However I.30E-DGEBA dispersions present different behavior, and after being subjected to the curing protocol they do not show any exothermic reaction, and only a $T_{g}$ is detected around $120^{\circ} \mathrm{C}$. This confirms that under the curing protocol the epoxy homopolymerization is promoted by the $\mathrm{I} .30 \mathrm{E}$.

To analyze the effect of the clay on the $T_{g}$ of the cured epoxy networks, nanocomposites with C93A were prepared using DDM following the curing protocol specified in Sec. 2.2. For all the compositions studied a single $T_{g}$ at high temperature was observed, which corresponds to the cured epoxy network. No residual heat of reaction was detected indicating that the epoxy had been totally cured. Table 3 presents the values of $T_{g}$ of the cured nanocomposites, as can be seen second and third scans lead to slightly higher $T_{g}$ values due to the postcuring that takes place during the previous scan. The $T_{g}$ values of cured epoxy gradually decrease with increasing the clay content. This can be attributed to the plasticization of the crosslinked epoxy networks by the hydrocarbon chains of the alkyl ammonium ions [12]. A reduction of $T_{g}$ is also expected if non-perfect networks are formed due to stoichiometric imbalances originated by the selective intercalation of epoxy and DDM [21].

In comparison with previous results it can be concluded that for nanocomposites with low clay content $(\leq 6 \mathrm{wt} \%)$ there are no significant differences in $T_{g}^{\prime} s$ of samples having the same content of C93A, C30B, or $\mathrm{C} 15 \mathrm{~A}$ [18]. However the $T_{g}$ of I.30E/epoxy nanocomposites was clearly lower (see Table 3). This is consistent with the hypothesis that $\mathrm{I} .30 \mathrm{E}$ induces the epoxy homopolymerization even on curing with DDM and the network formed is a less crosslinked epoxy.

TABLE $3 T_{g}$ of the Samples Cured in an Oven (1hour $-120^{\circ} \mathrm{C}$ and 2hours $-180^{\circ} \mathrm{C}$ ) Obtained from the 1st, 2nd, and 3rd DSC Scans

\begin{tabular}{lccc}
\hline & \multicolumn{3}{c}{$T_{g}\left({ }^{\circ} \mathrm{C}\right)$} \\
\cline { 2 - 4 } wt\% & 1st scan & 2nd scan & 3rd scan \\
\hline C93A & & & \\
0 & 163 & 167 & 168 \\
2 & 158 & 164 & 165 \\
4 & 157 & 162 & 163 \\
6 & 154 & 159 & 160 \\
$\mathbf{1 0}$ & 151 & 156 & 157 \\
$\mathbf{1 2}$ & 146 & 152 & 153 \\
$\mathbf{1 . 3 0 E}$ & & & \\
2 & 154 & 160 & 161 \\
4 & 148 & 153 & 154 \\
6 & 147 & 152 & 153 \\
\hline
\end{tabular}




\subsection{WAXD and TEM of Clay/Epoxy Nanocomposites}

Figure 3(a) shows the WAXD patterns of C93A/epoxy nanocomposites that were cured under the protocol described in Sec. 2.2. The neat C93A shows an intense peak at $2 \theta=3.44^{\circ}$ that corresponds to an interlayer distance $d_{001}=2.56 \mathrm{~nm}$. The nanocomposites show the $d_{001}$ basal diffraction peaks at lower $\theta$ values, the peaks at $2 \theta \sim 2.6^{\circ}$ corresponding to an interlayer distance of $d_{001} \sim 3.35 \mathrm{~nm}$ which is higher than in neat C93A. This indicates that C93A/epoxy nanocomposites are intercalated, because for a fully exfoliated clay it is expected the absence of peaks in WAXD pattern, $\left(1.5^{\circ}<2 \theta<10^{\circ}\right)$ and $d$-spacing of at least $6 \mathrm{~nm}[12]$.
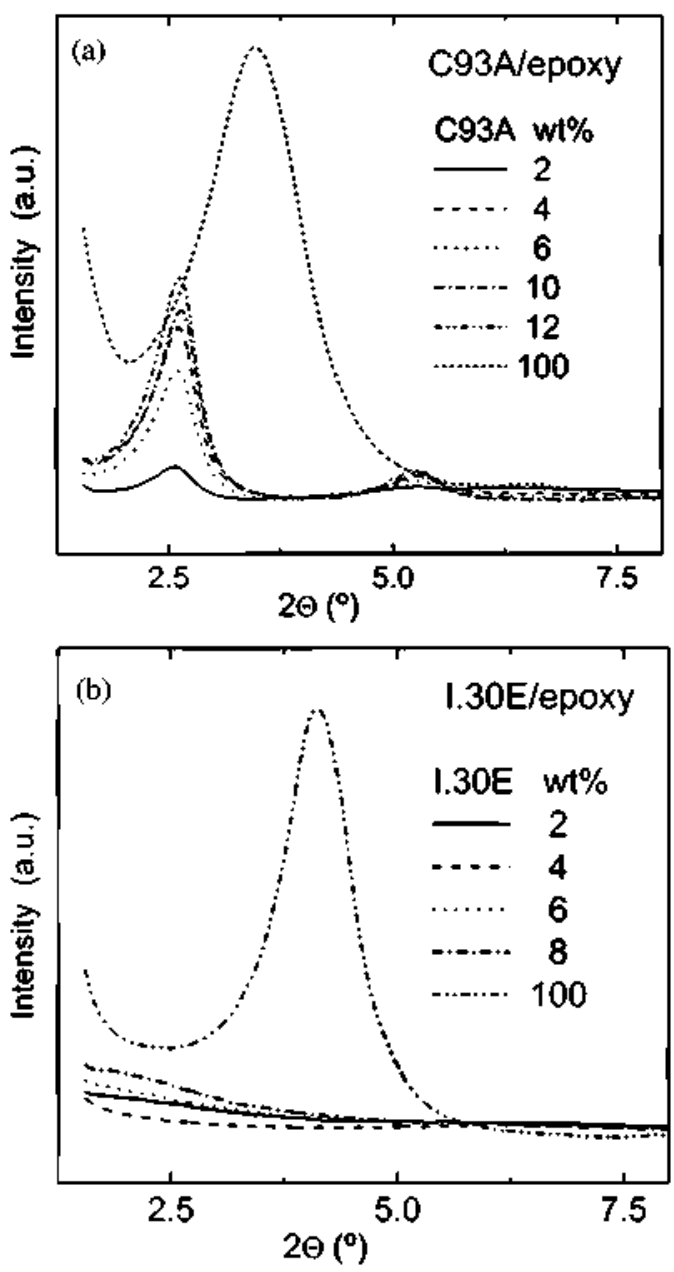

FIGURE 3 WAXD patterns of (a) C93A and C93A/epoxy nanocomposites and (b) I.30E and I. $30 \mathrm{E} /$ epoxy nanocomposites. 
TABLE $4 d_{001}$ for C93A/Epoxy, C30B/Epoxy, and C15A/Epoxy Nanocomposites

\begin{tabular}{cccc}
\hline & \multicolumn{3}{c}{$d_{\text {iw1 }}(\mathrm{nm})$} \\
\cline { 2 - 4 } w\% clay & $\mathrm{C} 93 \mathrm{~A}$ & $\mathrm{C} 30 \mathrm{~B}$ & $\mathrm{C15 \textrm {A }}$ \\
\hline 2 & 3.41 & 3.47 & 3.47 \\
4 & 3.35 & 3.49 & 3.41 \\
6 & 3.37 & 3.51 & 3.38 \\
10 & 3.30 & - & - \\
12 & 3.33 & - & 3.15 \\
100 & 2.56 & 1.82 & \\
\hline
\end{tabular}

Table 4 collects the values of $d_{001}$ for the C93A/epoxy nanocomposites. It can be noticed that $d_{001}$ slightly decreases as the C93A content increases, an effect that was previously observed [18]. Table 4 also includes $d_{001}$ values for $\mathrm{C} 30 \mathrm{~B} /$ epoxy and $\mathrm{C} 15 \mathrm{~A} /$ epoxy nanocomposites previously reported [18]. The $d$-spacings in C30B/epoxy and C15A/epoxy nanocomposites $\left(d_{001} \sim 3.5 \mathrm{~nm}\right.$ and $\sim 3.4 \mathrm{~nm}$, respectively) indicate that the Cloisite/epoxy nanocomposites present intercalated structures. The increment of interlayer distances $\Delta d_{001}$ are 1.58 for $\mathrm{C} 30 \mathrm{~B}, 0.74$ for $\mathrm{C} 93 \mathrm{~A}$, and 0.25 for $\mathrm{C} 15 \mathrm{~A}$, hence epoxy molecules intercalate less in C15A than in $\mathrm{C} 30 \mathrm{~B}$ and $\mathrm{C} 93 \mathrm{~A}$. The organophilic nature of the alkyl ammonium Cloisites makes it possible for epoxy monomers to access into the clay galleries [1-5] and pushes the silicate layers apart forming intercalated structures. The cation exchange capacity (content of alkyl ammonium ions) is higher for $\mathrm{C} 15 \mathrm{~A}$ than for $\mathrm{C} 93 \mathrm{~A}$ and $\mathrm{C} 30 \mathrm{~B}$ (see Table 1), moreover the alkyl ammonium cations in C15A and C93A have two alkyl chains (hydrogenated tallow), whereas $\mathrm{C} 30 \mathrm{~B}$ cations have only one alkyl chain. It can be concluded that more space is occupied by the alkyl ammonium ions in C15A galleries, and there is less intragallery space available for epoxy monomers than in $\mathrm{C} 93 \mathrm{~A}$ and $\mathrm{C} 30 \mathrm{~B}$. As a consequence the epoxy molecules intercalate in a smaller proportion in C15A than in C93A and C30B.

It is interesting to notice that the three Cloisites reach in the nanocomposites similar interlayer spacing, $d_{001} \sim 3.35 \mathrm{~nm}$ for C93A, $d_{001} \sim 3.5 \mathrm{~nm}$ for $\mathrm{C} 30 \mathrm{~B}$, and $d_{001} \sim 3.4 \mathrm{~nm}$ for $\mathrm{C} 15 \mathrm{~A}$. The alkyl chain lengths of the ammonium cations are similar for the three Cloisites (see Table 1) leading to similar intragallery spacing indicating a similar arrangement of the alkyl ammonium cations within the gallery space. This is consistent with a layer of inclined alkyl ammonium ions (paraffin-type) that is, the alkyl ammonium ions reorient into the Cloisite galleries forming parafin type layer that permits to accommodate the epoxy molecules $[6,8]$.

Figure 3(b) shows the WAXD patterns of I.30E/epoxy nanocomposites. No peak is observed which means that the interlayer spacing $d_{001}$ is larger than $6 \mathrm{~nm}$ then an exfoliated estructure can be assigned [12]. This is a consequence of the catalytic effect of the acidic alkyl ammonium cations in I.30E which favors intragallery polymerization of the epoxy and conduces to the clay 
exfoliation, in accordance with the Pinnavaia proposition [7]. However, the exfoliated structure does not imply a homogeneous dispersion of the clay layers in the epoxy matrix. In fact, clay tactoids retaining the parallel orientation of the platelets are detected in these nanocomposites as it is illustrated in the TEM micrographs of I.30E/epoxy and C93A/epoxy nanocomposites shown in Fig. 4. The analysis of the micrographs corresponding to I.30E/epoxy nanocomposite gives an average interlayer distance of $13 \pm 2 \mathrm{~nm}$.

\subsection{DMTA of Clay/Epoxy Nanocomposites}

Figure 5 shows the $\tan \delta$-temperature dependence for the C93A/epoxy nanocomposites. The $\tan \delta$ peak is the $\alpha$-relaxation associated to the glass transition, and is used as a measure of $T_{g}$. The temperature values of the $\tan \delta$ maximum decrease with increasing of the clay content. These results are in agreement with those obtained by DSC (see Table 3). Similar behavior was found for I.30E/epoxy nanocomposites.

Table 5 collects $\tan \delta$ peak data of C93A/epoxy and I.30E/epoxy nanocomposites, together with those of C30B/epoxy and C15A/epoxy nanocomposites previously reported [18]. All the nanocomposites show a reduction of $T_{g}$ that depends on the clay content. Two effects can contribute to lowering the epoxy $T_{g}$ : plasticizing effect of alkyl chains and imbalances of stoichiometry. The alkyl chains of the ammonium cations plasticize the epoxy
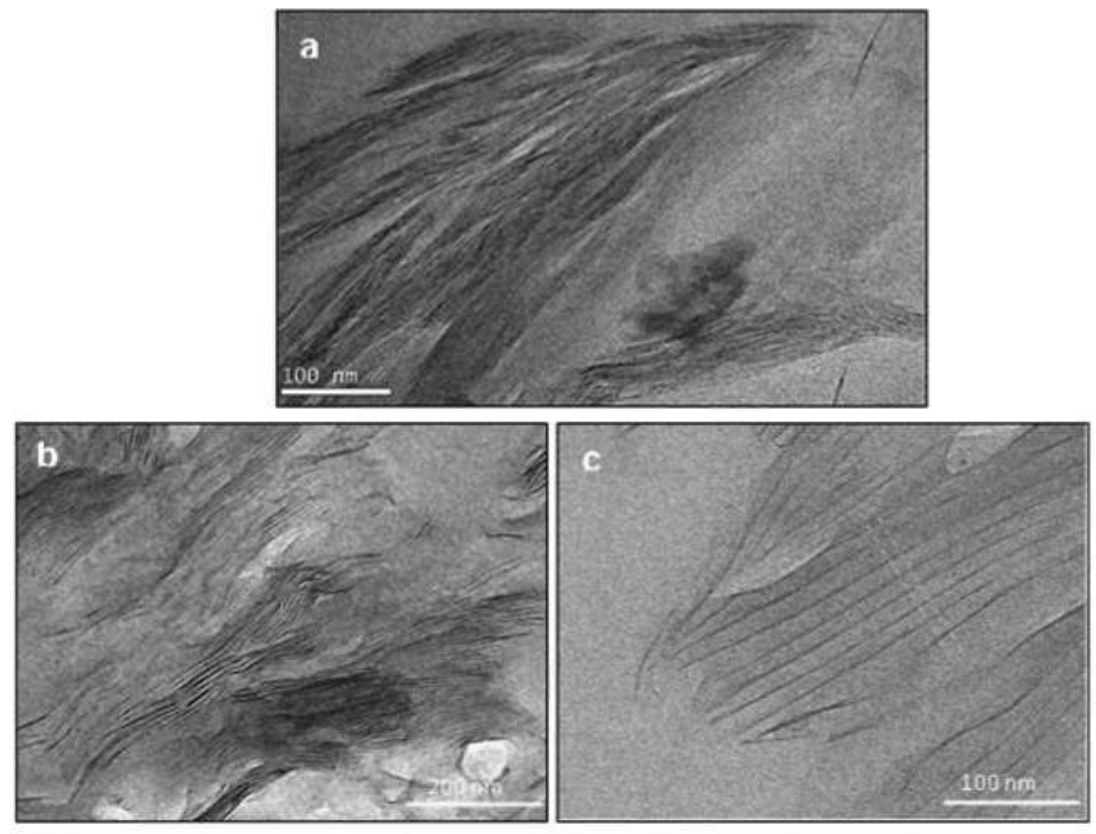

FIGURE 4 TEM micrographs of (a) C93A/epoxy, (b, c) I.30E/epoxy nanocomposites with different magnifications. 


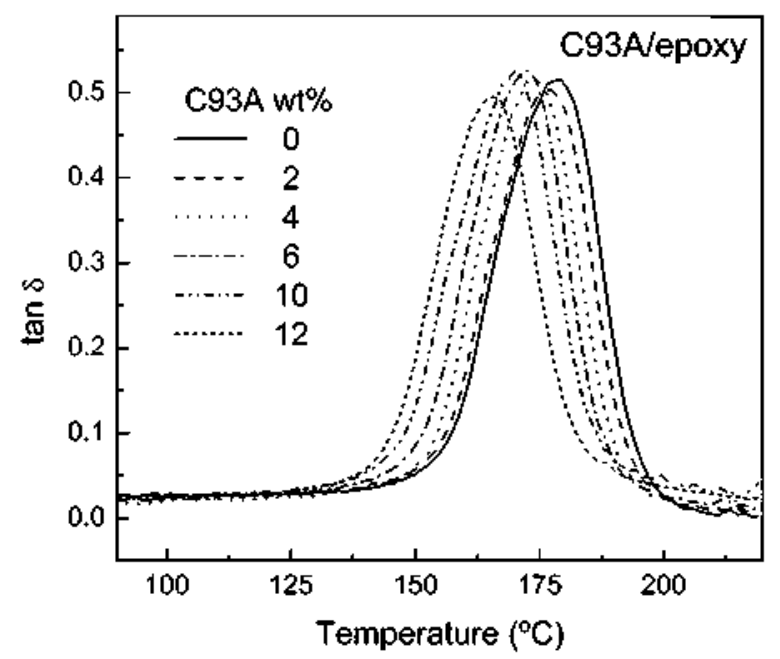

FIGURE $5 \tan \delta$-temperature dependence for C93A/epoxy nanocomposites.

thermoset, this effect would be favored by the interactions between epoxy and the organic chains. As we have discussed above, the three Cloisite nanocomposites: C93A/epoxy, C30B/epoxy, and C15A/epoxy have intercalated structures, and $\Delta d_{001}$ reflects that epoxy molecules intercalate in higher extension in C30B than in C93A, but only are slightly intercalated in C15A. Therefore C15A nanocomposites would present lower reduction of $T_{g}$ than C93A and then $\mathrm{C} 30 \mathrm{~B}$. Otherwise $\mathrm{C} 15 \mathrm{~A}$ and $\mathrm{C} 93 \mathrm{~A}$ have two long alkyl chains that will provide more free volume in the interaction with the epoxy network, thus resulting in higher reduction of $T_{g}$ than $\mathrm{C} 30 \mathrm{~B}$. A lower $T_{g}$ means that the segmental mobility of the network increases, thus the second effect that would contribute to decrease the $T_{g}$ would be the lowering of the epoxy crosslinking density. In intercalated nanocomposites the lowering of crosslinking density may be originated by imbalances of stoichiometry, as a consequence of preferential intercalation epoxy monomers, and the imbalance would be greater the larger the intercalation is $\mathrm{C} 30 \mathrm{~B}>\mathrm{C} 93 \mathrm{~A}>\mathrm{C} 15 \mathrm{~A}$. In $\mathrm{I} .30 \mathrm{E} /$ epoxy nanocomposites

TABLE $5 T_{g}$ Values from the $\tan \delta$ Maximum in DMTA Experiments for Cloisites/Epoxy and I.30E/Epoxy

\begin{tabular}{lcccc}
\hline & \multicolumn{5}{c}{$T_{g}\left({ }^{\circ} \mathrm{C}\right)$} \\
\cline { 2 - 5 } wt\% clay & C93A & C30B & C15A & I.30E \\
\hline 0 & 179 & 179 & 179 & 179 \\
2 & 176 & 176 & 174 & - \\
4 & 174 & 174 & 173 & 169 \\
6 & 173 & 175 & - & 167 \\
10 & 170 & - & - & - \\
12 & 166 & - & - & - \\
\hline
\end{tabular}




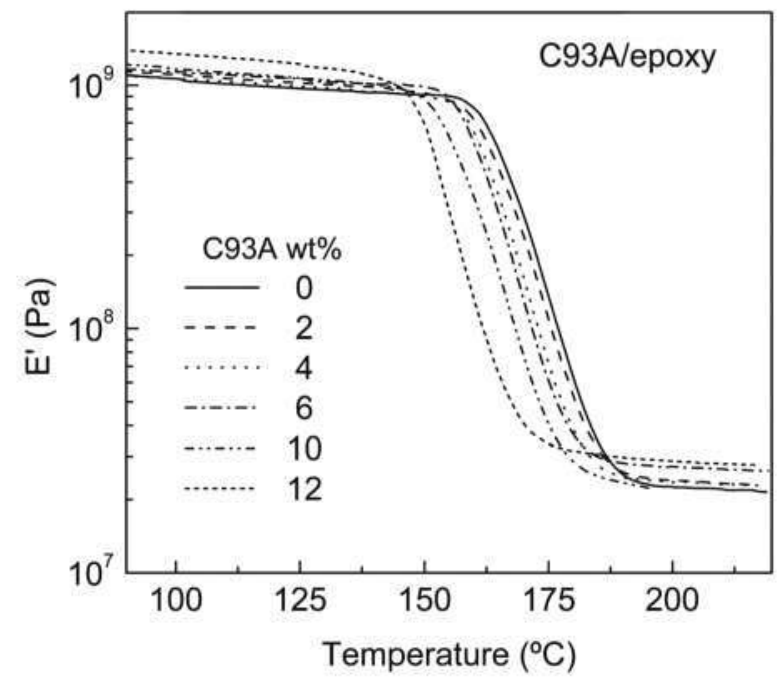

FIGURE 6 E'-temperature dependence for C93A/epoxy nanocomposites.

having an exfoliated structure, epoxy molecules are more exposed to the action of the organocations which favors its homopolymerization and the plasticizer action, thereby justifying the greatest decrease in $T_{g}$.

Figure 6 shows the elastic or storage modulus $\left(E^{\prime}\right)$ vs. temperature curves of $\mathrm{C} 93 \mathrm{~A} / \mathrm{epoxy}$ nanocomposites. The values of $E^{\prime}$ in the glassy state $\left(T<T_{g}\right)$ and in the rubbery state $\left(T>T_{g}\right)$ increase as the C93A content increases.

The relative values of storage modulus referred to the neat epoxy thermoset $\left(E_{\mathrm{r}}^{\prime}=\underline{E}_{\text {nanocomposite }}^{\prime} / E_{\text {epoxy }}^{\prime}\right)$ are shown in Figs. 7 and 8 , at $40^{\circ} \mathrm{C}$ (glassy state) and $200^{\circ} \mathrm{C}$ (elastomeric or rubbery state), respectively, for

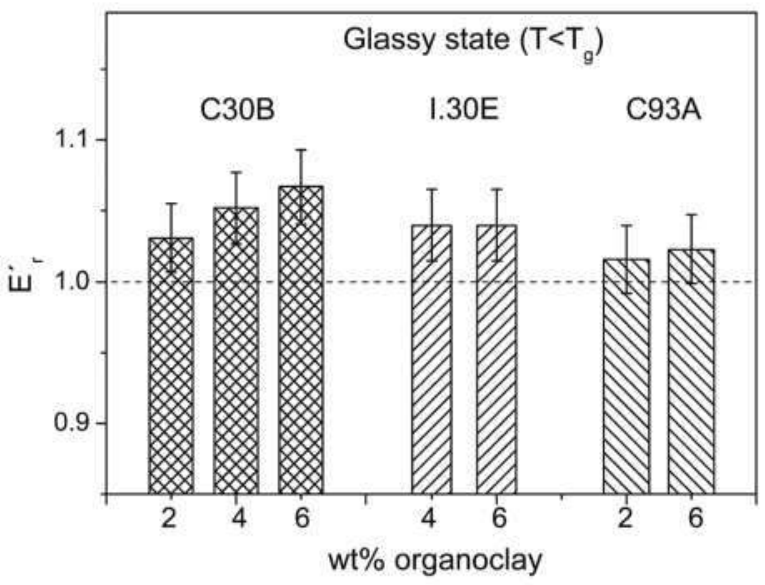

FIGURE $7 \mathrm{E}_{\mathrm{r}}^{\prime}=\mathrm{E}_{\text {nanocomposite }}^{\prime} / \mathrm{E}_{\text {cpoxy }}^{\prime}$ in the glassy state for C93A/epoxy and I.30E/epoxy nanocomposites along with $\mathrm{E}_{\mathrm{r}}^{\prime}$ calculated from previous results of $\mathrm{C} 30 \mathrm{~B} / \mathrm{epoxy}$ nanocomposites. 


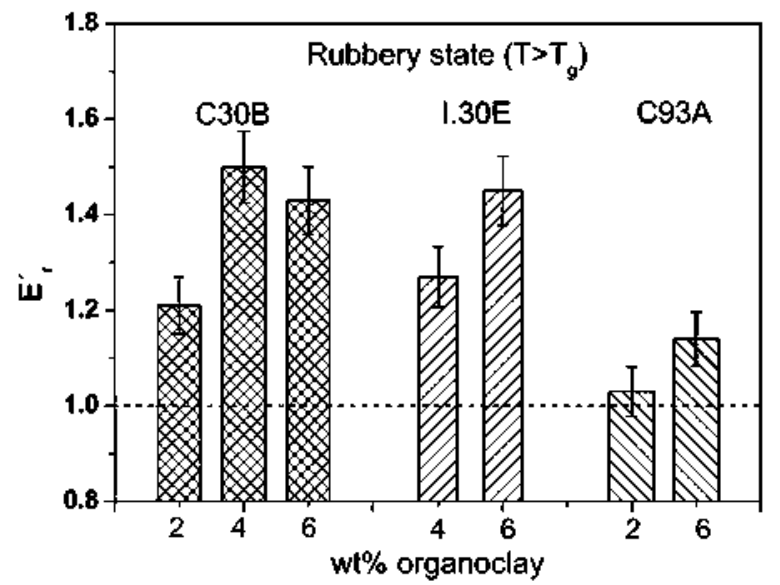

FIGURE $8 \mathrm{E}_{\mathrm{r}}^{\prime}=\mathrm{E}_{\text {nanocomposite }}^{\prime} \mathrm{E}_{\text {cpoxy }}^{\prime}$ in the rubbery state for $\mathrm{C} 93 \mathrm{~A} / \mathrm{epoxy}$ and $\mathrm{I} .30 \mathrm{E} / \mathrm{epoxy}$ nanocomposites along with $\mathrm{E}_{\mathrm{r}}^{t}$ calculated from previous results of $\mathrm{C} 30 \mathrm{~B} /$ epoxy nanocomposites.

nanocomposites C93A/epoxy and I.30E/epoxy along with those calculated from previous results of $\mathrm{C} 30 \mathrm{~B} / \mathrm{epoxy}[18]$. C30B/epoxy and I.30E/epoxy nanocomposites have storage modulus higher than the neat epoxy thermoset, especially in the elastomeric state. However increases of $E^{\prime}$ for C93A/ epoxy nanocomposites are less significant. C15A nanocomposites do not show rigidity improvements [18]. This behavior is clearly related to the level of intercalation of the clays, the greater the intercalation, the more effective the load transfer and higher the module. Note that it is difficult to increase the rigidity of a highly crosslinked thermoset (high $T_{g}$ ).

\subsection{Lap Shear Strength of Organoclay/Epoxy-Aluminum Joints}

To study the adhesive behavior of the organoclay/epoxy nanocomposites, the adhesive strength of organoclay/epoxy-aluminum joints was measured by single lap shear test. The experiments were performed in freshly prepared joints and joints aged by water immersion $\left(170\right.$ hours at $\left.75^{\circ} \mathrm{C}\right)$ and the results obtained are shown in Fig. 9 (mean values and standard deviation). The data were statistically analyzed by a Student $t$-test $(\alpha=0.05)$ resulting confidence intervals (95\% CI) in MPa: (18.1-15.0), (17.2-14.4), and (14.9-12.0) for neat epoxy, C30B/epoxy and I.30E/epoxy, respectively, and (9.1-6.7), (6.7-4.4), and (9.3-8.5) for water aged neat epoxy, C30B/epoxy, and I.30E/epoxy, respectively. Therefore freshly prepared joints with neat epoxy and with $\mathrm{C} 30 \mathrm{~B} /$ epoxy adhesives exhibited similar shear bond strength, while I.30E/ epoxy adhesives gave slightly lower strength. The shear strength for C93A/epoxy epoxy adhesive ( $\sigma=14.2 \pm 0.9 \mathrm{MPa}$ with confidence interval: $15.1-13.3 \mathrm{MPa}$ ) was intermediate between $\mathrm{C} 30 \mathrm{~B} / \mathrm{epoxy}$ and $1.30 \mathrm{E} /$ epoxy and the aging in water for joints using C93A/epoxy adhesive was not studied. 


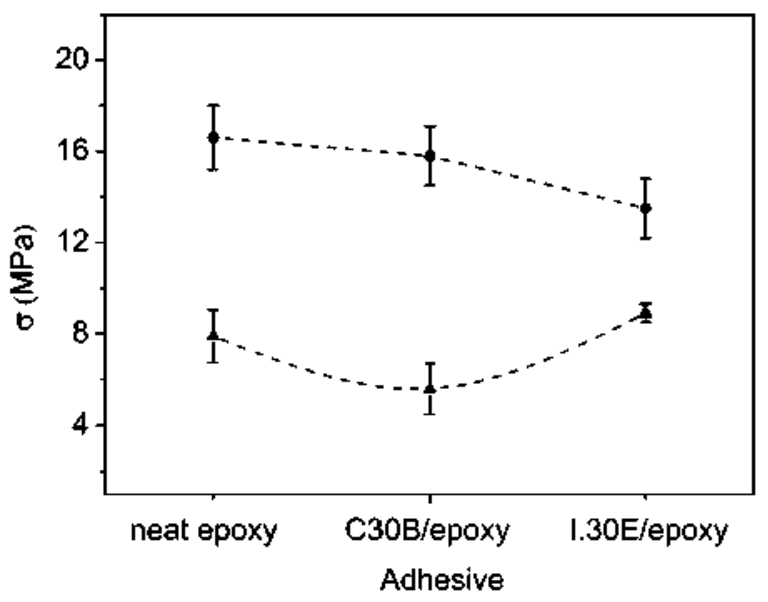

FIGURE 9 Adhesive strength (lap shear test) for neat epoxy, I.30E/epoxy and C30B/epoxy adhesives. Freshly prepared joints (๘) and joints aged in water $\left(75^{\circ} \mathrm{C}\right)(\boldsymbol{\Lambda})$.

It is known that aging in water weakens the joints, breaking the adhesive-adherent interface, and promoting adhesive failure. Even for treated aluminum surfaces the shear strength of the joints decreased $50-60 \%$ for neat epoxy and $\mathrm{C} 30 \mathrm{~B} /$ epoxy. However with I.30E/epoxy the strength loss is less than $30 \%$, these joints showed the highest strength after aging.

The justification for this behavior is based on the polarity of the adhesive, the higher the adhesive polarity the greater its interaction with the aluminum surface and the greater the work of adhesion and the joint strength [26]. The organoclays can modify the polarity of adhesive due to two opposing effects: increased polarity due to $\mathrm{OH}$ groups and decreased polarity by nonpolar alkyl chains of the organocations. The similar behavior of $\mathrm{C} 30 \mathrm{~B} /$ epoxy and neat epoxy adhesives suggests that in $\mathrm{C} 30 \mathrm{~B} /$ epoxy these effects may be compensated. The lower $T_{g}$ of the $\mathrm{C} 30 \mathrm{~B} /$ epoxy adhesive could justify the easier diffusion of water and the lower strength upon aging.

The lower initial strength of the joints with I.30E/epoxy adhesive can be attributed to the low polarity of the alkyl chains of the organocations, and their strength upon aging could be due to the impediment of the alkyl chains to the water diffusion, which also would be hindered by the exfoliated nanocomposite structure.

\section{CONCLUSIONS}

Two organically modified montmorillonites: C93A and I.30E were used as nanoreinforcements of an epoxy adhesive. The influence of the organoclays on the curing, thermal, and mechanical behavior of nanocomposites were studied and compared with those of C15A/epoxy and C30B/epoxy. 
Furthermore, the strength and durability of epoxy-aluminum joints have been studied using these adhesives.

The four clays accelerate epoxy homopolymerization and the curing reaction with diamines. The effect is related to the chemical nature of the organoclay cations being more intense in the order: $\mathrm{I} .30 \mathrm{E}>\mathrm{C} 30 \mathrm{~B}>\mathrm{C} 93 \mathrm{~A}>\mathrm{C} 15 \mathrm{~A}$.

The Cloisite nanocomposites (cured adhesives) have intercalated structures. The extent of intercalation of epoxy molecules between the clay platelets increases in the order: C30B/epoxy $>$ C93A/epoxy $>C 15 \mathrm{~A} /$ epoxy. The I.30E/epoxy nanocomposites present higher intercalation resulting exfoliated structures (separation between platelets $>10 \mathrm{~nm}$ ). This is consequence of the different chemical nature of the organocations.

The presence of organoclays decreases the $T_{g}$ of the epoxy adhesive, resulting $T_{g} \quad(\mathrm{I} .30 \mathrm{E} /$ epoxy $)<T_{g} \quad(\mathrm{C} 30 \mathrm{~B} /$ epoxy $) \sim T_{g} \quad(\mathrm{C} 93 \mathrm{~A} / \mathrm{epoxy}) \sim T_{g}$ (C15A/epoxy). This behavior is related to the clay intercalation and is explained considering two factors: stoichiometric imbalances that give rise to less crosslinked networks and plasticizing effect of the organocations.

The C30B/epoxy, C93A/epoxy, and I.30E/epoxy nanocomposites have higher storage modulus than pure epoxy thermoset, this improvement is not found in the C15A/epoxy nanocomposites. The module increases can be correlated with the extent of the intercalation.

The freshly prepared joints of aluminum substrates using I.30E/epoxy adhesives exhibit slightly lower shear strength than using neat epoxy and $\mathrm{C} 30 \mathrm{~B} /$ epoxy adhesives. In joints that were aged in water the strength decreases $50-60 \%$ for neat epoxy and $\mathrm{C} 30 \mathrm{~B} / \mathrm{epoxy}$ adhesives, while the strength loss for I.30E/epoxy adhesive is less than $30 \%$. These results can be justified by considering the polarity of the organocations and the intercalation of the organoclays.

\section{FUNDING}

The authors acknowledge financial support provided by Universidad Politécnica de Madrid (UPM Research Groups).

\section{REFERENCES}

[1] Pascault, J. P., Sautereau, H., Verdu, J., and Williams, R. J. J., Thermosetting Polymers, (Marcel Dekker, New York, 2002).

[2] Tjong, S. C., Mat. Sci. Eng. R. 53, 73-197 (2006),

[3] Betega de Paiva, L., Morales, A. R, and Valenzuela Díaz, F. R., Appl. Clay Sci. 42, 8-24 (2008).

[4] Pavlidou, S. and Papaspyrides, C. D., Prog. Polym. Sci. 33, 1119-1198 (2008).

[5] Azeez, A. A., Rhee, K. Y., Park, S. J., and Hui, D., Comp. Pant B. 45, 308-320 (2013) 
[6] Messersmith, P. B. and Giannelis, E. P., Chem. Mater. 6, 1719-1725 (1994).

[7] Lan, T., Kaviratna, P. D., and Pinnavaia, T. J., J. Phys. Chem. Solids. 57, $1005-1010$ (1996).

[8] Wang, Z. and Pinnavaia, T., Chem. Mater. 10, 1820-1826 (1998).

[9] Kornmann, X., Lindberg, H., and Berglun, L. A., Polymer. 42, 1303-1310 and $4493-4499$ (2001).

[10] Triantafillidis, C. S., Le Baron, P. C., and Pinnavaia, T. J., J. Solid State Chem. 167, 354-362 (2002).

[11] Xu, W., Bao, S., and He, P., J. App. Polym. Sci. 84, 842-849 (2002).

[12] Park, J. H. and Jana, S. C., Macromolecules. 36, 8391-8397 (2003).

[13] Le PLuart, L., Duchet, J., and Sautereau, H., Polymer. 46, 12267-12278 (2005).

[14] Wang, L, Wang, K., Chen, L., Zhang, Y., and He, C., Comp. Part. A. 37, 1890-1896 (2006).

[15] Román, F., Monserrat, S., and Hutchinson, J. M., J. Therm. Anal. Calorim. 87, $113-118(2007)$.

[16] Ingram, S. E., Liggat, J. J., and Pethrick, R. A., Polym. Int. 56, 1029-1034 (2007).

[17] Xidas, P. I. and Triantafyllidis, K. S., Eur. Polym. J. 46, 404 417 (2010).

[18] Prolongo, M. G., Martínez-Casado, F. J., Masegosa, R. M., and Salom, C., J. Nanosci. Nanotech. 10, 2870-2879 (2010).

[19] Wang, Y., Zhang. B., and Ye, J., Mater. Sci. Eng. A 528, 7999-8005 (2011).

[20] Ngo, T. D., Ton-That, M. T., Hoa, S. V., and Cole, K. C., Polym. Eng. Sci. 52. 607-614 (2012).

[21] García del Cid, M. A., Prolongo, M. G., Salom, C., Arribas, C., SánchezCabezudo, M., and Masegosa, R. M., J. Therm. Anal. Calonim. 108, 741-749 (2012).

[22] Hutchinson, J. M., Shiravand, F., and Calventus, Y., J. Appl. Polym. Sci. 128, 2961-2970 (2013).

[23] Al-Safy, R., Al-Mahaidi, R., Simon, G. P., and Habsuda, J., Constr. Buil. Mater. 28, 769-778 (2012).

[24] Russel, W. J. and Garnis, E. A., US Patent No. 4,212,701, published 15 July 1980.

[25] Prolongo, S. G., Horcajo, K. F., Del Rosario, G., and Ureña, A., J. Adthes. Sci. Tecbnol. 24, 1097-1112 (2010).

[26] Kinloch, A. J., Adbesion and Adhesives, (Chapman and Hall, New York, 1987). Ch. 3, p. 83 . 